\title{
Univ. Prof. Dr. Karlheinz Schenk 1925-2012
}

\author{
(C) Springer-Verlag Wien 2012
}

Vor wenigen Tagen erreichte uns die traurige Nachricht, dass Herr Univ. Prof. Dr. Karlheinz Schenk am Samstag, dem 23. Juni 2012 nach langer schwerer Krankheit im 87. Lebensjahr verstorben ist. Am 12. Juli 2012 fand er seine letzte Ruhestätte auf dem Friedhof Wien Heiligenstadt.

Dr. Karlheinz Schenk wurde am 17. Oktober 1925 in Wien geboren. Sein Vater Dr. Karl Schenk war Lehrer und Hauptschuldirektor und mit seiner Gattin Louise verheiratet. Nach dem Realgymnasium in 1030 Wien wurde er vorzeitig zum Wehrdienst einberufen, verbrachte seine Militärzeit bei den Gebirgsjägern und wurde 1945 zum Leutnant befördert. Nach dem Italienfeldzug und der Kapitulation der Südarmee konnte er sich nach Fulpmes in Tirol durchschlagen und wurde in den letzten Kriegstagen aus dem Heer entlassen.

1954 heiratete Dr. Schenk seine Gattin Friederike, die er schon vor vielen Jahren nach schwerer Krankheit verloren hat. 1964 wurde sein Sohn Wolfgang geboren.

Im Oktober 1945 begann er sein Medizinstudium in Wien und promovierte am 15. Juni 1950 an der Alma Mater Rudolfina zum Dr. univ. med. Bereits im Juli 1950 trat Dr. Schenkin die I. Univ. Augenklinik unter Prof. Pillat ein, der er Zeit seines Lebens bis zur Pensionierung treu geblieben ist. 1954 wurde er klinischer Assistent (= Oberarzt), habilitierte 1961 und wurde 1975 zum a.o. Universitäts-Professor ernannt. Jahrelang leitete Prof. Schenk an der I. Univ. Augenklinik die Station 19 mit der Kin-

Wien, im Juli 2012 derabteilung. Nach der Emeritierung Prof. Pillats folgte 1964-1981 Prof. Hruby als Klinikchef. Nach dessen Emeritierung wurde Prof. Schenk zum supplierenden Leiter der Augenklinik für zwei Jahre ernannt. Nach über 40-jähriger Tätigkeit an der I. Univ. Augenklinik trat er am 31. Dezember 1990 in den Ruhestand.

Neben zahlreichen Vorträgen vor der ÖOG, der DOG und der Ophthalmologischen Gesellschaft in Wien publizierte Prof. Schenk an die 80 wissenschaftliche Arbeiten, die überwiegende Anzahl davon als Alleinautor. Er machte sich auch einen Namen als Gutachter. Neben dieser klinischen und wissenschaftlichen Tätigkeit führte Prof. Schenk eine Privatpraxis und war als exzellenter Operateur bekannt und gesucht. 1968-1995 gehörte Prof. Schenk dem Vorstand der Österreichischen Ophthalmologischen Gesellschaft an. Jahrzehntelang war er einer meiner Vorgänger im Amt des Kassiers der ÖOG. In diese Zeit fällt auch das Erbe von Frau Dr. A. Rabensteiner, welches Prof. Schenk gewinnbringend anzulegen verstanden hat.

Prof. Schenk war ein ausgezeichneter Diagnostiker, ein gerechter Prüfer und beliebter Vortragender in seinen Vorlesungen. Wir jüngeren Kollegen haben von ihm sehr viel lernen können. Er pflegte ein freundschaftliches Verhältnis zu seinen Fachkollegen und war ein beliebter Unterhalter bei gesellschaftlichen Abenden. Wir werden ihn in ehrendem Angedenken bewahren.

MR. Prim. Prof. Dr. Paul Drobec 